the proof of Theorem 1 . The result follows since distinct subsequences yield disjoint subspaces of $W$.

Added in proof. With a slight modification $U$ can also be universal in the Mioduszowski continuous image sense. The point $(0,0, \cdots)$ of $U$ determined by the inverse limit representation is an endpoint and thus we can add a pseudo-arc $P$ such that $P \cup U$ is snake-like. If $X$ is any snake-like continuum, let $f$ map $P$ onto $X$ by [2] and extend $f$ to $P \cup U$ by collapsing $U$ to the appropriate point.

\title{
BIBLIOGRAPHY
}

1. Morton Brown, Some applications of an approximation theorem for inverse limits, Proc. Amer. Math. Soc. 11 (1960), 478-483.

2. J. Mioduszowski, A functional conception of snake-like continua, Fund. Math. 51 (1962), 179-189.

Louisiana State University

\section{A NOTE ON COUNTABLY PARACOMPACT SPACES AND METRIZATION}

\author{
C. E. AULL
}

As defined by Dowker [1], a topological space $(X, J)$ is called countably paracompact if every countable open cover of $X$ has an open locally finite refinement. It is known that every metrizable topological space is paracompact and hence countably paracompact.

It is proposed to show that in the usual metrization theorem for topological spaces $\left[3\right.$, p. 127], the $T_{3}$ condition may be replaced by the combination $T_{2}$ and countably paracompactness. We will need the following lemma.

LeммA. Every countably paracompact, first countable $T_{2}$ space is $T_{3}$.

Proof. Designate the topological space by $(X, J)$ as in Kelley [3]. Let $F$ be a proper closed set of $X$. For $x \notin F$, and $y \in F$ there exists disjoint open set $U_{x}^{(y)}$ and $U_{y}$ containing $x$ and $y$ respectively. There exists a countable base at $x,\left\{V_{k}\right\}, k=1,2, \cdots, n, \cdots$. Let $W_{k}=U\left\{U_{y}: U_{x}^{(y)} \supset V_{k}\right\} . W_{k} \cap V_{k}=\varnothing$ and $\left\{W_{k}\right\}$ forms a countable cover of $F$. Since $F$ is closed, $\left\{W_{k}\right\}$ may be replaced by an open locally finite refinement, $\left\{T_{a}: a \in A\right\}$ where $A$ is an index set.

Received by the editors December 28, 1964. 
Each $T_{a} \subset W_{k}$ for some $k$ so $x \notin \bar{T}_{a}$. Set $T=U\left\{T_{a}: a \in A\right\}$. $\bar{T}=U\left\{\bar{T}_{a}: a \in A\right\}$. Hence $\sim \bar{T}$ and $T$ are disjoint open sets containing $[x]$ and $F$ respectively and the lemma follows.

A topological space with a $\sigma$-locally finite base is first countable and we have the following theorem as a consequence of the usual metrization theorem.

TheOREM. A topological space with a $\sigma$-locally finite base is metrizable iff it is $T_{2}$ and countable paracompact.

One may be replace the $T_{2}$ condition by any condition which together with the first countable axiom imply that $T_{2}$ is satisfied, such as

(a) Every sequence converges in $(X, J)$ to at most one point in $(X, \Im)$.

(b) Every compact set is closed [2]

It might be noted that if (b) is satisfied in a topological space, (a) is also satisfied.

\section{BIBLIOGRAPHY}

1. C. H. Dowker, On countably paracompact spaces, Canad. J. Math. 3 (1951), 219-224.

2. E. Halfar, A note on Hausdorff separation, Amer. Math. Monthly 68 (1961), 164.

3. J. L. Kelley, General topology, Van Nostrand, New York, 1955.

Kent State University 